\title{
Wave Packet Realization of Lightlike States ${ }^{\star}$
}

\author{
G. DOMOKos \\ Department of Physics, John Hopkins University Baltimore, Md., USA
}

Received November 2, 1971

\begin{abstract}
We construct a new realization of lightlike particle states. The realization considered corresponds to objects which form partially localized wave packets in the space dimensions transverse to the direction of the lightlike momentum. The states of the transverse motion are classified by the representations of an algebra $S U(1,1)$ constructed of the generators of the conformal group. It is conjectured that this realization is suitable for the formulation of the "parton"-model of hadrons.
\end{abstract}

\section{Introduction}

Lightlike particle states play an ever-increasing role in physics. Apart from classical objects (photons, neutrinos), the hypothesis has been recently put forward that hadrons may consist of elementary constituents and when viewed from a reference frame moving with - approximately the velocity of light, a hadron may well be represented as a "beam" of free "partons" [1].

With these applications in mind, we studied the various realizations of lightlike states. Clearly, from the mathematical point of view, this problem reduces to the study of the linear spaces spanned by all lightlike states on which a representation of a - physically reasonable - invariance group can be realized. Within the framework of the Poincare group, the problem has been solved in the classical paper by Wigner [2]. Intuitively one expects, however, that a lightlike state should be essentially unchanged (i.e. brought over into another lightlike state) by certain conformal transformations (uniform accelerations and dilations) as well. This turns out to be indeed the case. By studying these additional transformations one discovers a somewhat unusual and - to the present author's knowledge - hitherto unknown type of states. These have the interesting property that they are partially localized - they form "transverse wave packets" - in the two space dimensions perpendicular to the direction in which the "particle" moves with light velocity. (The "transverse space".) One may thus speculate that such a set of states is probably appropriate for the description of the "almost free" constituents of the hadrons and/or the physical hadron states themselves. The purpose of this paper is to exhibit the states just mentioned by means of an elemen-

* Research supported in part by the U.S. Atomic Energy Commission under Contract No. AT(30-1)-4076. 
tary algebraic construction and to study some of their properties, in particular, their physical interpretation.

After this investigation was finished, we learned that in an attempt to give a mathematically consistent formulation of the droplet model of hadrons, Gürsey and Orfanidis constructed a set of particle states [3] ${ }^{1}$, which for lightlike momenta are the same as the states described here.

The construction described in this paper seems to have the advantage that it can be easily generalized to quantum field theories with interaction $^{2}$. We are grateful to Professor Gürsey for communicating to us his results prior to publication and also for stimulating discussions.

This paper was completed during the author's stay at the Aspen Center for Physics. He wishes to thank the Center for the hospitality extended to him.

\section{Lightlike States and the Transformations which Leave them Invariant}

A lightlike single particle state without intrinsic spin is entirely characterized by its momentum, which - in a Cartesian basis - satisfies the condition:

$$
p_{0}=\left(p_{1}^{2}+p_{2}^{2}+p_{3}^{2}\right)^{\frac{1}{2}} .
$$

By a rotation we can always achieve that $p_{1}=p_{2}=0$. It is convenient to introduce a different basis (the "lightlike basis" or $L$-basis) in Minkowski space adapted to the standardized lightlike momentum $\left(p_{1}=p_{2}=0\right.$, $\left.p_{3}=p_{0}\right)$ as follows [4]. Define:

$$
\begin{aligned}
& p_{z}=\frac{1}{\sqrt{2}}\left(p_{0}+p_{3}\right) \equiv k, \\
& p_{t}=\frac{1}{\sqrt{2}}\left(p_{0}-p_{3}\right) \equiv h, \\
& \boldsymbol{p}=\left\{p_{1}, p_{2}\right\} .
\end{aligned}
$$

In the $L$-basis the nonvanishing components of the metric tensor, $g_{\mu v}$ are:

$$
\begin{aligned}
& g_{i k}=\delta_{i k} \quad(i, k=1,2), \\
& g_{z t}=g_{t z}=-1 .
\end{aligned}
$$

A general spinless lightlike state can be characterized by the numbers, $p, k$, and from (2.1) through (2.2) the fourth component of the momentum, $h$ satisfies:

$$
h=\frac{p^{2}}{2 k} \text {. }
$$

${ }_{1}$ Wave packet states have been known for some time for timelike and spacelike momenta, cf. F. Gürsey, report C NAEM 53, Istanbul, 1965; unpublished.

2 This is due to the circumstance that our construction proceeds via the generators of the conformal group, whereas the works quoted in Ref. [3] use position operators of the Newton-Wigner type. The generalization of the present construction to a quantum field theory with interaction will be considered in a separate paper. 
We shall call $k$ the color of the state (borrowing the name from the description of a light wave propagating in the 3-direction) and $p$ its transverse momentum.

Next, we list the commutation relations of the generators of the conformal group in the $L$-basis. It will be convenient to introduce special symbols for some of the generators. Let $M_{\mu \nu}, P_{\mu}, K_{\mu}, D$ denote the generators of homogeneous Lorentz transformations, translations, uniform accelerations and dilations, respectively, where $\mu, v$ run through the values $1,2, z, t$. Introduce the notation:

$$
\begin{aligned}
M_{z t} & =N, & M_{z i} & =E_{i} \\
M_{12} & =M, & M_{t i} & =F_{i} \\
P_{z} & =K, & P_{t} & =H
\end{aligned}
$$

(Operators will be denoted by capitals, the corresponding eigenvalues by lower case letters.)

The commutation relations in question read as follows:

$$
\begin{aligned}
& {\left[M, E_{i}\right]=i \varepsilon_{i j} E_{j} \quad\left[M, F_{i}\right]=i \varepsilon_{i j} F_{j}} \\
& {\left[N, E_{i}\right]=-i E_{i} \quad\left[N, F_{i}\right]=i F_{i}} \\
& {\left[E_{i}, E_{j}\right]=0 \quad\left[F_{i}, F_{j}\right]=0} \\
& {\left[E_{i}, F_{j}\right]=-i \delta_{i j} N+i \varepsilon_{i j} M} \\
& {\left[E_{i}, P_{j}\right]=i \delta_{i j} K \quad\left[F_{i}, P_{j}\right]=i \delta_{i j} H} \\
& {\left[M, P_{i}\right]=i \varepsilon_{i j} P_{j} \quad\left[N, P_{i}\right]=0} \\
& {\left[E_{i}, K\right]=0 \quad[M, K]=0} \\
& {\left[F_{i}, K\right]=i P_{i} \quad[N, K]=-i K} \\
& {\left[F_{i}, H\right]=0 \quad[M, H]=0} \\
& {\left[E_{i}, H\right]=i P_{i} \quad[N, H]=i H} \\
& {\left[P_{\mu}, P_{v}\right]=0} \\
& {\left[K_{i}, P_{j}\right]=-2 i\left(\delta_{i j} D+\varepsilon_{i j} M\right)} \\
& {\left[K_{i}, K\right]=2 i E_{i} \quad\left[K_{i}, H\right]=2 i F_{i}} \\
& {\left[K_{i}, K\right]=0 \quad\left[K_{z}, H\right]=2 i(D-N)} \\
& {\left[K_{z}, P_{i}\right]=-2 i E_{i}\left[K_{t}, P i\right]=-2 i F_{i}} \\
& {\left[K_{t}, H\right]=0 \quad\left[K_{t}, K_{i}\right]=2 i(D+N)} \\
& {\left[D, P_{\mu}\right]=-i P_{\mu} \quad\left[D, K_{\mu}\right]=i K_{\mu}} \\
& {\left[M_{\mu v}, D\right]=0 \quad\left[M_{\mu v}, K_{\varrho}\right]=i\left(g_{v \varrho} K_{\mu}-g_{\mu \varrho} K_{v}\right)} \\
& {\left[K_{\mu}, K_{v}\right]=0 .}
\end{aligned}
$$


Here $\varepsilon_{i j}$ is the Levi-Civitá tensor in two dimensions. The group (2.5a) involves the commutators of the Poincare algebra only, whereas those in group (2.5b) contain the generators $K_{\mu}, D$ as well.

A standard, spinless, lightlike state is defined by:

$$
\begin{aligned}
\boldsymbol{P}_{i}|k\rangle & =0, \\
K|k\rangle & =k|k\rangle, \\
W_{z}|k\rangle & =0, \\
W^{2}|k\rangle & =0,
\end{aligned}
$$

where $W$ is the Wigner operator (the generator of the little group), $W^{2}=W_{\mu} W^{\mu}$.

We are going to look for transformations which leave the color invariant, i.e. for the - linear combinations of - generators which commute with $K$. By inspecting the commutation relations (2.5), we find that the operators satisfying this requirement are the following:

$$
P_{i}, H, M, E_{i}, K_{z}, A=D-N .
$$

(Had we considered the group of commutators (2.5a) only, the last two operators would have been left out; they are those additional operators we mentioned in the introduction.)

The commutators of the operators with each other can be read off from (2.5). Evidently, $E_{i}, M$ span the algebra of $E(2)$, generating the Wigner little group of the standard momentum. The operators (2.7) have the property that they commute with $K$ even if the state is non-standard. Evidently this is a generalization of the concept of a little group.

One verifies with the help of (2.5) that the operators $H, K_{z}, A$ form a sub-algebra which is isomorphic to $S U(1,1)$. In order to be able to construct dimensionsless operators, one has to introduce a characteristic energy, say $\omega$. The existence of a characteristic energy is an intrinsic property of the representations considered. It is easy to check that the linear combinations:

$$
J_{1}=-\frac{1}{\omega} H-\frac{\omega}{2} K_{z}, \quad J_{2}=A, \quad J_{3}=\frac{1}{\omega} H-\frac{\omega}{2} K_{z}
$$

satisfy the commutation relations of $S U(1,1)$ in the standard form, viz.:

$$
\begin{aligned}
& {\left[J_{1}, J_{2}\right]=-i J_{3}} \\
& {\left[J_{3}, J_{1}\right]=i J_{2}} \\
& {\left[J_{2}, J_{3}\right]=i J_{1} .}
\end{aligned}
$$

In the next section we construct a realization of the algebra of the operators $(2.7)$ (based on a discrete representation of $S U(1,1)$ ) which seems to have a particular intuitive appeal. 


\section{Construction of the Transverse Wave Packet States}

The algebra spanned by the generators (2.7) can be realized on the universal enveloping algebra of the operators (2.5a). Indeed, the commutator between $E_{i}$ and $P_{i}$ suggests to introduce the operator:

$$
X_{i}=K^{-1} E_{i}=E_{i} K^{-1}=\frac{1}{K} E_{i},
$$

which satisfies:

$$
\left[X_{i}, P_{j}\right]=i \delta_{i j} .
$$

(We exclude states with $k=0$.)

Notice further that the kinematics in the transverse space is Galilean [5]: $M, E_{i}, P_{i}, A$ generate the direct product of the Galilei group in two dimensions with dilatations. (The operator $H$ and $K$ play the role of the "Hamiltonian" and the "mass", respectively.) Therefore $X_{i}$ can be called the position operator in transverse space.

One readily verifies that the operators:

$$
\begin{gathered}
E_{i}=K X_{i}, \quad M=-\varepsilon_{i j} P_{i} X_{j}, \\
K_{z}=-K \boldsymbol{X}^{2}, \quad A=-\frac{1}{2}(\boldsymbol{X} \boldsymbol{P}+\boldsymbol{P} \boldsymbol{X}), \\
H=\frac{\boldsymbol{P}^{2}}{2 K}
\end{gathered}
$$

realize the algebra spanned by the generators (2.7); the abstract generators and their realizations (3.3) are denoted by the same symbols. The intuitive meaning of this realization becomes evident if one observes that the operator

$$
\omega J_{3}=\frac{\boldsymbol{P}^{2}}{2 K}+\frac{1}{2} K \omega^{2} \boldsymbol{X}^{2}
$$

acts on the lightlike states of color $k$ just as the "Hamiltonian" of a nonrelativistic two dimensional oscillator does. The vectors in the space of lightlike states belonging to this particular realization can be conveniently labelled by the eigenvalues of $J_{3}, K$ and $M$. In order to see this, one has to resort only to the apparatus of elementary quantum mechanics. Introduce the operators $\boldsymbol{B}, \boldsymbol{B}^{\dagger}$ by the relations:

$$
\begin{aligned}
& \boldsymbol{X}=\frac{1}{\sqrt{2 K \omega}}\left(\boldsymbol{B}+\boldsymbol{B}^{\dagger}\right), \\
& \boldsymbol{P}=i \sqrt{\frac{K \omega}{2}}\left(\boldsymbol{B}^{\dagger}-\boldsymbol{B}\right),
\end{aligned}
$$


which diagonalize $J_{3}$ :

$$
J_{3}=\frac{1}{2}\left(\boldsymbol{B}^{\dagger} \cdot \boldsymbol{B}+\boldsymbol{B} \cdot \boldsymbol{B}^{\dagger}\right) .
$$

The operator $M$ will be diagonalized by going over to a "spherical" basis, viz.

$$
B_{ \pm}=\frac{1}{\sqrt{2}}\left(B_{1} \mp i B_{2}\right), \quad B_{ \pm}^{\dagger}=\frac{1}{\sqrt{2}}\left(B_{1}^{\dagger} \pm i B_{2}^{\dagger}\right) .
$$

In this basis the generators of the subalgebra $S U(1,1)$ and $M$ are written as follows:

$$
\begin{aligned}
& J_{1}=\left(B_{+}^{\dagger} B_{-}^{\dagger}+B_{+} B_{-}\right), \\
& J_{2}=i\left(B_{+} B_{-}-B_{+}^{\dagger} B_{-}^{\dagger}\right), \\
& J_{3}=\left(B_{+}^{\dagger} B_{+}+B_{-}^{\dagger} B_{-}+1\right), \\
& M=B_{+}^{\dagger} B_{+}-B_{-}^{\dagger} B_{-} .
\end{aligned}
$$

The states so characterized will be denoted by $\left|k j_{3} m\right\rangle$. Eigenstates of the position and momentum operators are coherent states of the fictious oscillator. For example, one immediately verifies that

$$
\boldsymbol{P}(\exp i \boldsymbol{p} \boldsymbol{X})|k, 00\rangle=\boldsymbol{p} \exp i \boldsymbol{p} \boldsymbol{X}|k, 00\rangle .
$$

The representation of the "quasispin" algebra (3.6) can be readily extended to a representation to the full stability algebra of the color, (2.7), by means of the relations (3.3), (3.4) and remembering that the states $\left|k j_{3} m\right\rangle$ can be constructed by

$$
\left|k j_{3} m\right\rangle=\frac{\left(B_{+}^{\dagger}\right)^{\frac{1}{2}\left(j_{3}+m-1\right)}\left(B_{-}^{\dagger}\right)^{\frac{1}{2}\left(j_{3}-m-1\right)}}{\left[\left(\frac{j_{3}+m-1}{2}\right) !\left(\frac{j_{3}-m-1}{2}\right) !\right]^{\frac{1}{2}}}|k\rangle .
$$

The calculation of the matrix elements of the operators (2.7) can be done in an elementary way using the commutators

$$
\left[B_{m}, B_{m^{\prime}}^{+}\right]=\delta_{m m^{\prime}}, \quad\left[B_{m}, B_{m^{\prime}}\right]=\left[B_{m}^{+}, B_{m^{\prime}}^{+}\right]=0 \quad\left(m, m^{\prime}= \pm\right) .
$$

We shall not go through this elementary exercise, but point out that the states (3.7) span a reducible, infinite spin representation of the integer type of the Wigner little group. This can be seen by noticing that the Casimir, $\boldsymbol{E}^{2}$, is not an invariant of (2.7) and the operators $E_{i}$ are not nilpotent.

\section{Orthogonality and Completeness}

In order to construct a suitable scalar product, we need the representation of a generator affecting $k$. Choose for instance the generator $N$. 
Using (2.5), (3.3) we establish the relations:

$$
[N, \boldsymbol{P}]=[N, \boldsymbol{X}]=0 .
$$

Further, using (2.5) and the identity:

$$
0=\left[N, K K^{-1}\right]=K\left[N, K^{-1}\right]+[N, K] K^{-1},
$$

we derive:

$$
\left[N, K^{-1}\right]=i K^{-1} .
$$

This gives the commutators:

$$
\begin{aligned}
& {\left[N, J_{3}\right]=-i J_{1},} \\
& {\left[N, J_{2}\right]=0,} \\
& {\left[N, J_{1}\right]=-i J_{3} .}
\end{aligned}
$$

This shows that $N$ acts on the quasispin as $-J_{2}$. Using (2.5) again (notice that $N$ acts on the color as a dilatation operator), we finally get:

$$
N\left|k j_{3} m\right\rangle=\left(-i k \frac{\partial}{\partial k}-J_{2}\right)\left|k j_{3} m\right\rangle .
$$

Thus we find that the orthogonality and completeness relations (with respect to which the operators (3.3), (3.10) are self-adjoint) read:

$$
\sum_{j_{3} m} \int \frac{d k}{k}\left|k j_{3} m\right\rangle\left\langle k j_{3} m\right|=\mathbf{1}, \quad\left\langle k j_{3} m \mid k^{\prime} j_{3}^{\prime} m^{\prime}\right\rangle=k \delta\left(k-k^{\prime}\right) \delta_{j_{3} j_{3}} \delta_{m m^{\prime}} .
$$

It is easy to extend these considerations to representations of the halfinteger type of the little group. Notice that the "ground state" of the fictious oscillator in (3.7) is a lightlike state with standard momentum and vanishing intrinsic spin. One could, however, choose instead any state transforming according to a finite spin representation of the Wigner little group to build up the "tower" (3.7). Let such a state be $|k, \lambda\rangle(\lambda=0, \pm 1 / 2, \ldots)$.

Based on these states, one builds up towers:

$$
\left|k j_{3} m \lambda\right\rangle=\frac{\left(B_{+}^{\dagger}\right)^{\frac{1}{2}\left(j_{3}+m-\lambda-1\right)}\left(B_{-}^{\dagger}\right)^{\frac{1}{2}\left(j_{3}-m+\lambda-1\right)}}{\left\{\left[\frac{1}{2}\left(j_{3}+m-\lambda-1\right)\right] !\left[\frac{1}{2}\left(j_{3}-m+\lambda-1\right)\right] !\right\}^{\frac{1}{2}}}|k \lambda\rangle .
$$

The analog of the realization (3.3) is:

$$
M=-\varepsilon_{i j} \boldsymbol{P}_{i} \boldsymbol{X}_{j}+\lambda
$$

the other operators being unchanged. 


\section{Physical Interpretation and Discussion}

The physical interpretation of the states just constructed involves elementary calculations not going beyond the apparatus of a first course in quantum mechanics. Indeed, one verifies immediately that the projection of $\left|k j_{3} m \lambda\right\rangle$ onto an eigenstate of the transverse position or mementum operator, e.g. $\left\langle k \boldsymbol{x} \mid k j_{3}\right\rangle$, is just the Schrödinger wave function of a two dimensional harmonic oscillator. Therefore these projections are proportional - both in coordinate and momentum space - to a Gaussian multiplied by an associated Laguerre polynomial; thus these states form wave packets of "minimum uncertainty" compatible with the given quantum numbers. (Hence the terminology used in Section 3.) This indeed was the starting point of the work of Gürsey and Orfanidis [3], referred to in the Introduction. The typical spread of the wave packet is governed by the characteristic energy scale, $\omega$, we have introduced.

In order to see this point more clearly, let us consider briefly the reduction properties of product representations. Consider e.g. a "twoparticle" system. The operators (3.3) now become direct sums of single particle operators:

$$
\begin{aligned}
E & =K^{(1)} \boldsymbol{X}^{(1)}+K^{(2)} \boldsymbol{X}^{(2)} \\
M & =-\varepsilon_{i j} P_{i}^{(1)} X_{j}^{(1)}-\varepsilon_{i j} P_{i}^{(2)} X_{j}^{(2)},
\end{aligned}
$$

etc. By introducing the operator analogs of the Jacobi coordinates, viz.:

$$
\begin{aligned}
\boldsymbol{P}^{(1)} & =\frac{K^{(1)}}{K^{(1)}+K^{(2)}} P+Q, & \boldsymbol{P}^{(2)} & =\frac{K^{(2)}}{K^{(1)}+K^{(2)}} P-Q, \\
\boldsymbol{X}^{(1)} & =X+\frac{K^{(2)}}{K^{(1)}+K^{(2)}} R, & \boldsymbol{X}^{(2)} & =X-\frac{K^{(1)}}{K^{(1)}+K^{(2)}} \boldsymbol{R}, \\
K & =K^{(1)}+K^{(2)}, & Q & =\frac{K^{(1)} \cdot K^{(2)}}{K^{(1)}+K^{(2)}},
\end{aligned}
$$

one splits the representation into what - in the language of quantum mechanics - corresponds to the motion of the center of mass and the relative motion. It can be shown by an elementary calculation that in a mixed representation, (where the center of mass is in a momentum eigenstate) the size of the wave packet of the relative motion is governed by the same scale, $\omega$, as in the single particle case. This circumstance provides a further motivation for the existence of the characteristic energy, $\omega$, Indeed, had we attempted to construct dimensionless "oscillator operators" by taking e.g.

$$
\pm \frac{1}{K} H-\frac{1}{2} K K_{z}
$$


these simple reduction properties of the product states would have been lost entirely.

We emphasize that the classification of this new type of states becomes possible only due to the existence of the enlarged stability group of the color; had we restricted ourselves to Lorentz transformations alone, the wave packet states could have not been given a simple algebraic description.

Two immediate generalizations of the construction given here seem to be possible. First, in view of an equivalence theorem proved by Leutwyler et al. [6], there should be no difficulty in including a rest mass parameter. Second, one can study the representations of the stability algebra of the color based on other realizations of the subalgebra $S U(1,1)$. It would be interesting to see whether these generalizations have as simple a physical interpretation as the states described here.

\section{References}

1. Feynman, R.P.: Phys. Rev. Letters 23, 1415 (1969).

2. Wigner, E.P.: Ann. Math. 40, 149 (1939).

3. Gürsey, F., Orfanidis, S.: Yale preprint (1972); unpublished.

4. Leutwyler, H.: Acta Phys. Austr. Supp. V. (1968). - Bardakci, K., Halpern, M.B.: Phys. Rev. 176, 1686 (1968). - Bacry, H., Chang, N.P.: Ann. Phys. (N.Y.) 47, 407 (1968). - Rohrlich, F.: Syracuse rep. (1970); unpublished. - Kogut, J. B., Soper, D.E.: Phys. Rev. D 1, 2901 (1970).

5. This has been emphasized by L. Susskind, Phys. Rev. 165, 1535 (1968).

6. Leutwyler, H., Klauder,J.R., Streit, L.: Nuovo Cimento 66 A, 536 (1970).

G. Domokos

The John Hopkins University

Dept. of Physics

Homewood Campus

Baltimore, Maryland 21218/USA 